\title{
Host response to coagulase-negative staphylococci in abscesses induced within mice
}

\author{
J. P. LONG and F. A. KAPRAL*
}

Department of Medical Microbiology and Immunology, The Ohio State University, Columbus, Ohio 43210-1239, USA

\begin{abstract}
Summary. A model whereby a known number of coagulase-negative staphylococci were packed into capillary tubes and implanted into the peritoneal cavity of mice proved to be a satisfactory method for generating abscesses that could be easily removed free of extraneous host tissue, and that permitted measurement of the survival of the organisms and accumulation of lipid in the lesion. Strains of S. epidermidis, S. schleiferi and S. lugdunensis, differing in their ability to produce fatty acid modifying enzyme (FAME) and lipase, were packed into either glass or plastic capillary tubes and used to generate abscesses. Abscesses produced by $S$. aureus served as comparators. Lipids accumulated within the abscesses caused by $S$. aureus in the same manner as previously described for the organism inoculated without tubes. ${ }^{1}$ Lipids also accumulated within abscesses produced by all the coagulase-negative staphylococci, but the rate of accumulation was slower and the lipid droplets were smaller than seen with $S$. aureus. The mobilisation of lipid did not differ in response to cocci in plastic or glass tubes. Strains of $S$. epidermidis and $S$. schleiferi producing FAME and lipase were better able to survive within abscesses than strains unable to produce these enzymes. However, FAME and lipase production did not appear to be the sole determinants of survival within abscesses. Regardless of whether they produced FAME and lipase, the two $S$. epidermidis strains were significantly better able to survive within plastic tubes than in glass tubes. No such difference was seen with $S$. aureus between plastic and glass tubes.
\end{abstract}

\section{Introduction}

A decade ago, we first detected a novel host defence mechanism which occurred in focal lesions caused by Staphylococcus aureus. ${ }^{2}$ We now know that this mechanism is dependent upon the production by the host of two types of bactericidal lipids - a pool of monoglycerides and a pool of long-chain unsaturated free fatty acids. Subsequently we found that $S$. aureus can produce an enzyme that can inactivate both bactericidal lipids. ${ }^{3}$ Called fatty acid modifying enzyme (FAME), it can esterify the fatty acids directly or it can transfer the acyl group of the monoglycerides to an appropriate alcohol. Produced by $80 \%$ of wild-type $S$. aureus strains, FAME is a virulence factor and only FAME-producing strains seem capable of surviving within the tissues. ${ }^{3,4}$

FAME is strongly inhibited by triglycerides, and the host produces a large pool of triglycerides within abscesses to inactivate the enzyme. To relieve this inhibition, $S$. aureus produces lipase that degrades the triglycerides. In addition, the lipase can inactivate the monoglycerides directly. ${ }^{2}$

Currently about 28 species of staphylococci other

Received 23 Sept. 1992; accepted 21 Jan. 1993.

* Correspondence should be sent to Professor F. A. Kapral. than S. aureus, grouped together as coagulase-negative staphylococci (CNS), have been described. Infections caused by some of these CNS are associated with implanted plastic prosthetic devices such as heart valves, cardiac pacemakers, artificial joints, ventricular shunts, vascular grafts, indwelling intra-venous catheters and peritoneal dialysis catheters. The reason why these plastic foreign bodies predispose to infections with CNS is not known.

CNS were tested for the production of FAME and lipase, and S. schleiferi and S. saprophyticus were found to most closely resemble $S$. aureus in that $80 \%$ of the strains produced both enzymes. ${ }^{3}$ In contrast, strains of $S$. lugdunensis and $S$. haemolyticus did not produce these enzymes and eight of 10 strains of $S$. simulans produced FAME but only one strain produced lipase. Among the other species, the proportion of strains producing both enzymes ranged from $10 \%$ to $60 \%$. Subsequently we wished to discover whether the host would mobilise the system of lipids against these organisms and whether FAME and lipase might be important for the survival of the organisms in vivo.

In previous studies abscesses were generated by injecting $S$. aureus directly into the peritoneal cavity, but for the present studies, a different infection model had to be used. In the case of $S$. aureus, the initial clumping reaction is essential to protect the inoculum 
from phagocytes and to allow abscess development. ${ }^{5,6}$ Most of the CNS do not possess clumping factor and, therefore, cannot be injected directly into the peritoneal cavity without being quickly phagocytosed. Therefore, the technique of Lambe and co-workers was modified; they implanted CNS pre-adhered to a plastic body. ${ }^{7}$ In this study a known number of organisms was packed into glass or plastic capillary tubes to prevent dissemination of the inoculum and to allow abscess formation. This then permitted measurement of the survival of the organisms and assessment of lipid accumulation in the lesions.

\section{Materials and methods}

\section{Staphylococcal strains}

Cultures of $S$. epidermidis, S. schleiferi and $S$. lugdunensis were obtained from either Dr J. Fluerette at the National Staphylococcal Reference Center in Lyons, France, or from Dr L. Ayers at the University Hospital, Ohio State University. Most strains had been isolated from closed space lesions.

$S$. epidermidis strain N860372 produced both FAME and lipase and strain N860081 produced neither enzyme. ${ }^{8} S$. lugdunensis strain N860410 produced neither FAME nor lipase. S. schleiferi strain N850388 produced FAME and lipase and strain N860265 produced neither enzyme. S. aureus I8Z, previously shown to produce FAME and lipase, was used as a reference strain.

\section{Slime production}

All staphylococci were evaluated for their ability to produce slime according to the method of Christensen et al. ${ }^{9}$ Briefly, organisms were first grown in trypticase soy broth (TSB) at $37^{\circ} \mathrm{C}$ for $24 \mathrm{~h}$ with agitation and then diluted 1 in 200 in $10 \mathrm{ml}$ of fresh TSB in conical polystyrene tubes. After incubation at $37^{\circ} \mathrm{C}$ for $48 \mathrm{~h}$, the tubes were emptied and stained with trypan blue to visualise adherent organisms.

\section{Staphylococcal suspensions}

Overnight cultures in TSB were centrifuged, washed and resuspended in TSB-saline (saline with TSB $8 \%$ $\mathrm{v} / \mathrm{v}$ ) to obtain a final concentration in excess of $1.5 \times 10^{10}$ organisms $/ \mathrm{ml}$. Suspensions were placed in plastic ampoules and stored at $-70^{\circ} \mathrm{C}$ until needed.

\section{Capillary tubes}

Glass microhaematocrit tubes (non-heparinised), $75 \mathrm{~mm}$ in length and with an internal diameter of $1 \mathrm{~mm}$, were obtained from Becton, Dickinson and Co, Parsippany, NJ, USA. A 1-mm column of liquid within the tubes corresponded to a volume of $0.78 \mu \mathrm{l}$.

Non-heparinised plastic tubing (PVC Drum Cartridge Catheter) with an internal diameter of $1.1 \mathrm{~mm}$ was obtained from Abbott Laboratories, Chicago, IL.
The siliconised needle was removed and the tubing was cut into $50-60-\mathrm{mm}$ sections before use. The volume in the lumen was $0.95 \mu \mathrm{l} /$ linear $\mathrm{mm}$.

Sterile plastic and glass capillary tubes were filled with an amount of suspension sufficient to contain $10^{9}$ cocci. Sterile gelatin ( $33 \%$ ) was placed into the lumen at one end to create a $5-10-\mathrm{mm}$ column adjacent to the cell suspension. The end with gelatin was then plugged with plasticine and the tubes were refrigerated for 5-10 min to allow the gelatin to solidify. The tubes were centrifuged aseptically in a haematocrit centrifuge to sediment the cocci against the gelatin layer. With a diamond point or scalpel blade, the tubes were cut through the centre of the gelatin layer and again above the top of the packed cell column to give a total length of 8-15 mm. The preparations were kept chilled in sterile petri dishes until used (within $1 \mathrm{~h}$ ).

\section{Survival of staphylococci}

Mice were anaesthetised with ether and the capillary tubes containing the packed cocci were implanted into the peritoneal cavity by inserting them through a 13 gauge needle used as a trocar. Mice were killed 5, 10 and 15 days after implantation and the abscesses were removed. Abscesses were trimmed of any adherent fat and were then homogenised in a tissue homogeniser. Abscesses containing glass tubes were first crushed in a sterile mortar and pestle before homogenising; abscesses containing plastic tubes were cut into $1-\mathrm{mm}$ sections before transferring to the homogeniser. All samples were homogenised in a total volume of $10 \mathrm{ml}$ of TSB-saline. Homogenates were serially diluted and cocci were enumerated by plate counts. With each experiment, representative capillary tubes (not implanted) containing packed cocci were homogenised and counts were done to verify the number of organisms originally present.

\section{Histological examination}

At each sample time some abscesses were removed and fixed in neutral buffered formalin. After fixation the capillary tube was removed through one end of the abscess and longitudinal frozen sections were cut and stained with either haematoxylin and eosin (H\&E) or Oil Red $\mathrm{O}$ to demonstrate lipids. ${ }^{10}$ Plastic and glass tubes, prepared in the same manner but lacking organisms, were also implanted intraperitoneally (i.p.). These non-infected controls were removed after 5,10 or 15 days, fixed in buffered formalin, and examined as before.

\section{Results}

\section{Slime production}

To avoid unnecessary complications in interpretation of results, all strains used in this study were evaluated for slime production; none produced slime. 


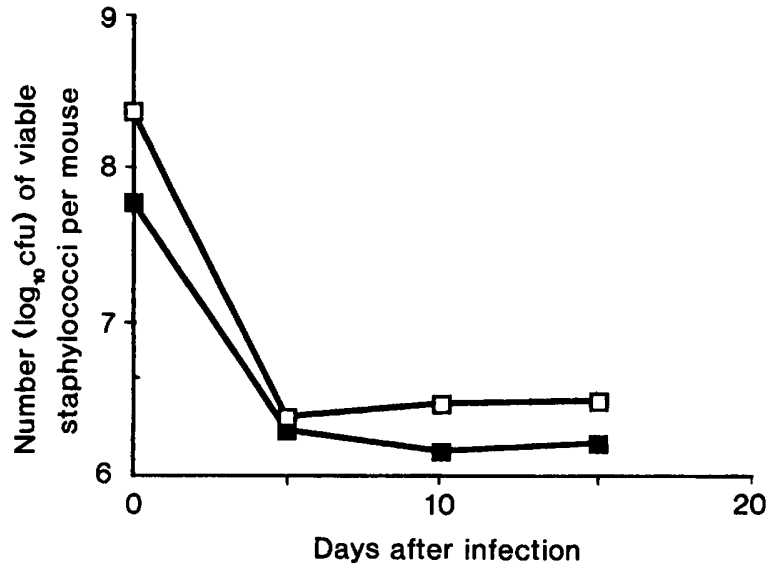

Fig. 1. The survival of $S$. aureus strain $18 \mathrm{Z}$ in glass (- - ) and plastic $(-\square-)$ capillary tubes implanted into the peritoneal cavity of mice. Values are geometric means.

\section{Survival of S. aureus}

In both plastic and glass capillary tubes, the populations of $S$. aureus strain $18 \mathrm{Z}$ decreased during the first 5 days but then remained relatively constant for the next 10 days (fig. 1). Histological examination revealed the usual tissue architecture expected in abscesses and showed the typical large accumulations of lipid beneath the connective tissue capsule. ${ }^{1,6}$ No obvious differences in the amount or rate of lipid accumulation were noted between lesions around plastic or glass tubes.

Implanted bacteria-free glass and plastic tubes were devoid of any organised abscess structure or lipid accumulations when removed from mice over the 15 day period of study.

\section{Survival of S. epidermidis}

When S. epidermidis strain N860372, that produced both FAME and lipase, was incorporated in glass tubes, it was continuously eliminated throughout the 15-day period of observation, but the decline was minimal after day 10 (fig. 2). However, within plastic tubes the population decreased for only the first 5 days, and thereafter no further decline occurred. Histological examination showed that lipid accumulated in abscesses around both the plastic and glass tubes, but the lipid droplets were smaller in size and slower to appear than in the response seen with $S$. aureus $18 \mathrm{Z}$ (fig. 3). The rate and extent of lipid mobilisation did not differ between abscesses containing plastic or glass tubes.

The viability of $S$. epidermidis strain N860081, that produced neither FAME nor lipase, was continuously reduced throughout the 15-day period in the glass tubes. On the other hand, the organisms lost viability in plastic tubes for the first 10 days, but then the population stabilised (fig. 2). However, in each case, this FAME- and lipase-negative strain survived less well than did the FAME- and lipase-producing $S$. epidermidis strain. Histological examination revealed a similar degree of lipid accumulation in abscesses

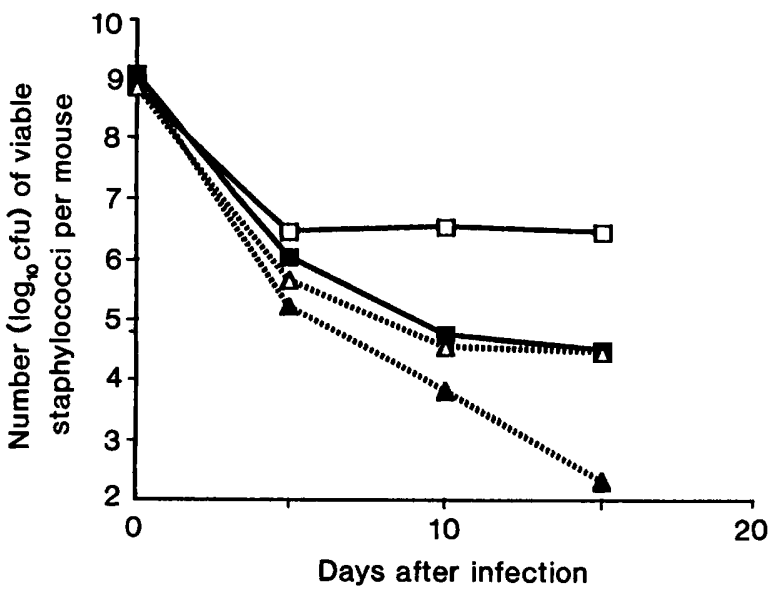

Fig. 2. The survival of $S$. epidermidis strain $\mathrm{N} 860372$ that produced both FAME and lipase $(\square, \square)$, and strain N860081 that produced neither enzyme $(\boldsymbol{\Delta}, \Delta)$, in glass $(\boldsymbol{\square}, \boldsymbol{\Delta})$ and plastic $(\square, \Delta)$ capillary tubes implanted in the peritoneal cavity of mice. Values are geometric means.

around glass and plastic tubes, but the mobilisation was nonetheless reduced and slower than that seen in abscesses caused by $S$. aureus strain $18 \mathrm{Z}$.

Statistical evaluation showed that both the $S$. epidermidis strain that produced FAME and lipase and the strain that lacked these enzymes were significantly better able to survive in plastic tubes than in glass tubes ( $\mathrm{p}$ values at 10 days were $<0.001$ and $<0.01$ respectively, at 15 days $<0.001$ ). Furthermore, the strain that produced both enzymes was also significantly better able to survive in plastic tubes than was the strain that lacked the enzymes ( $\mathrm{p}$ value at 10 days was $<0.001$, at 15 days $<0.01$ ).

\section{Survival of S. schleiferi}

Two S. schleiferi strains, one that produced both FAME and lipase and the other that produced neither enzyme, were continuously eliminated over the 15-day observation period when implanted i.p. within glass tubes (fig. 4). However, the FAME- and lipaseproducing strain survived significantly better than did the other strain $(\mathrm{p}<0.01$ at day $5,<0.001$ at days 10 and 15). Histological sections revealed typical abscess development, but the accumulation of lipids for both strains was slower and less in amount than in the host response to $S$. aureus strain $18 \mathrm{Z}$.

\section{Survival of $S$. lugdunensis}

A strain of $S$. lugdunensis, that produced neither FAME nor lipase, was continuously eliminated over a 15-day period within glass tubes (fig. 5). During the first 5 days the population decreased by about $\log _{10} 3$, but thereafter the remaining population decreased much more slowly. Histological examination of abscesses from 5 and 15 days revealed accumulations of lipid droplets, but again the droplets were smaller and the mobilisation was slower than in the host response to $S$. aureus. 

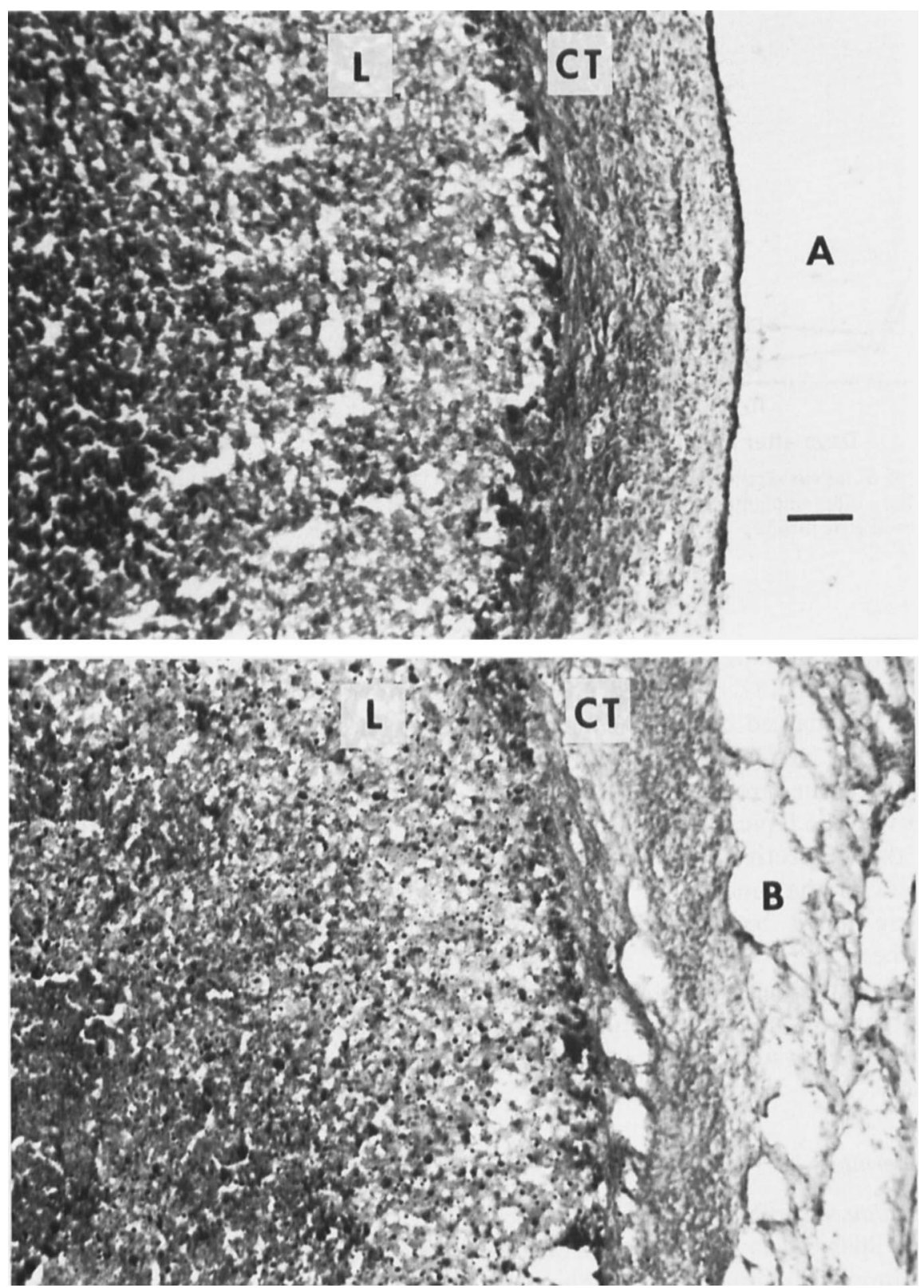

Fig. 3. Histological sections through 15-day-old abscesses generated by $S$. epidermidis strain $\mathrm{N} 860372$ in glass (A) and plastic (B) tubes. Sections were stained with Oil Red O to reveal lipid and photographed through a green filter to enhance contrast. Lipid droplets appear black: $\mathrm{CT}$, connective tissue capsule; L, leucocyte layer. The core of the lesion is towards the left. Bar, $100 \mu \mathrm{m}$.

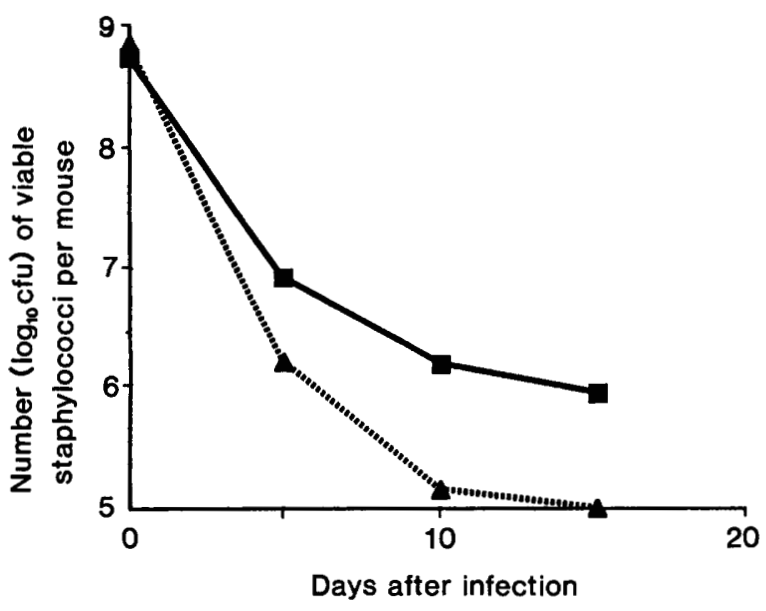

Fig. 4. The survival of $S$. schleiferi strain N850388 that produced both FAME and lipase (-n-) and strain N860265 that produced neither enzyme (-- - - ), in glass capillary tubes implanted in the peritoneal cavity of mice. Values are geometric means.

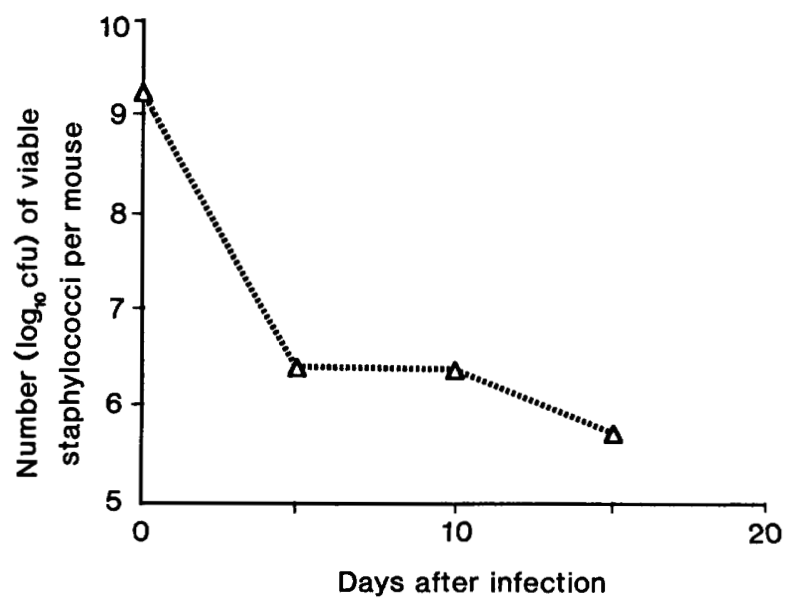

Fig. 5. The survival of $S$. lugdunensis strain N850410, in glass capillary tubes implanted in the peritoneal cavity of mice. This strain produced neither FAME nor lipase. Values are geometric means. 


\section{Discussion}

The infection model used in the present study involved packing a known number of organisms into either plastic or glass capillary tubes and inserting them into the peritoneal cavity of mice. This model provided a satisfactory means of creating abscesses with CNS approximating those produced by a similar number of $S$. aureus. The resultant abscesses could be easily removed free of extraneous host tissues, and the survival of the staphylococcal population and the accumulation of lipids could be monitored readily.

The i.p. injection of $S$. aureus resulted in the formation of abscesses with characteristic structural features. After 4 days the abscesses consist of a core of densely packed cocci, a broad zone of acellular debris, a zone of disintegrating leucocytes, a region of intact leucocytes, and a vascularised connective tissue capsule. ${ }^{6}$ Lipid accumulations were greatest immediately beneath the connective tissue capsule, but these accumulations broke up and small droplets moved through the deeper layers into the core of the lesion where the cocci were located. ${ }^{1}$ The implantation of CNS packed in plastic and glass capillary tubes resulted in the formation of abscesses very similar to those seen with $S$. aureus. Histological examination of CNS abscesses confirmed the overall cellular architecture and lipid accumulation normally seen with $S$. aureus abscesses (fig. 3).

In the case of $S$. aureus, the lipid appears to be brought into the lesion by lipid-laden macrophages first arriving several hours after abscess formation is initiated (Liu and Kapral, unpublished findings). Although lipid accumulation occurred in CNSinduced abscesses, the process was slower and the size of the lipid droplets was smaller than in abscesses produced by $S$. aureus packed in tubes. Whether the slower mobilisation of lipid by CNS resulted from a less vigorous signal to attract lipid-laden macrophages or represented an entirely different mech-

\section{References}

1. Shryock TR, Dye ES, Kapral FA. The accumulation of bactericidal lipids in staphylococcal abscesses. $J$ Med Microbiol 1992; 36: 332-336.

2. Kapral FA. A novel host defense mechanism controlling the survival of Staphylococcus aureus in focal lesions. In: Jeljaszewicz J, Ciborowski S (eds) The staphylococci. New York, Fischer Verlag. 1991: 69-76.

3. Mortensen JE, Shryock TR, Kapral FA. Modification of bactericidal fatty acids by an enzyme of Staphylococcus aureus. J Med Microbiol 1992; 36: 293-298.

4. Kapral FA, Smith S, Lal D. The esterification of fatty acids by Staphylococcus aureus fatty acid modifying enzyme (FAME) and its inhibition by glycerides. $J$ Med Microbiol 1992; 37: 235-237.

5. Kapral FA. Clumping of Staphylococcus aureus in the peritoneal cavity of mice. J Bacteriol 1966; 92: 1188-1195. anism for lipid accumulation, remains to be determined. It is also necessary to identify and quantify the individual lipids being accumulated, and compare these with lipids being accumulated with $S$. aureus.

The rate and amount of lipid mobilised versus CNS appeared to be the same in abscesses produced by the organism in plastic and glass tubes. Although the nature of the tubes did not appear to affect lipid accumulation, the two S. epidermidis strains packed in plastic tubes were significantly better able to survive in abscesses than when packed in glass tubes. No such disparity was seen in the survival of $S$. aureus packed in tubes. The mechanism whereby plastic affects survival of the organism is not known.

According to our current concepts regarding $S$. aureus host-parasite interactions, FAME and lipase are important virulence factors if the organism is to be able to survive within host tissues. ${ }^{2}$ Production of one enzyme without the other is of little avail to the organism. In a previous study of $86 \mathrm{CNS}$ strains encompassing 11 species, we found a high incidence of FAME- and lipase-producing strains in some species, but a low incidence in other species. ${ }^{8}$ Generally, however, there was a very strong correlation between FAME and lipase production, as would be expected if these enzymes were essential virulence factors in at least some species of CNS. In the present study, FAME- and lipase-producing strains of $S$. epidermidis and $S$. schleiferi were significantly better able to survive in abscesses than corresponding FAME- and lipasenegative strains. Although the results could be unrelated to the production of these enzymes, these findings, coupled with the observation that the host mobilises lipid in response to their presence, suggest that further study is necessary to determine whether the enzymes and the corresponding host defence mechanism operates in some CNS infections.

This investigation was supported by Bremer Foundation Grant \#9224.

6. Kapral FA, Godwin JR, Dye ES. Formation of intraperitoneal abscesses by Staphylococcus aureus. Infect Immun 1980; 30: 204-211.

7. Lambe DW, Ferguson KP, Gemmell CG, Keplinger JL. Pathogenic studies on five species of coagulase-negative staphylococci : a mouse model with a foreign body implant. In: Wadström $\mathrm{T}$ et al. (eds) Pathogenesis of wound and biomaterial-associated infections. Stuttgart, Springer Verlag. 1990: 255-263.

8. Long JP, Hart J, Albers W, Kapral FA. The production of fatty acid modifying enzyme (FAME) and lipase by various staphylococcal species. J Med Microbiol 1992; 37:232-234.

9. Christensen GD, Simpson WA, Bisno AL, Beachey EH. Adherence of slime-producing strains of Staphylococcus epidermidis to smooth surfaces. Infect Immun 1982; 37: 318-326.

10. Culling CFA. Handbook of histopathological techniques, 2nd edn. Washington, DC, Butterworth. 1963. 